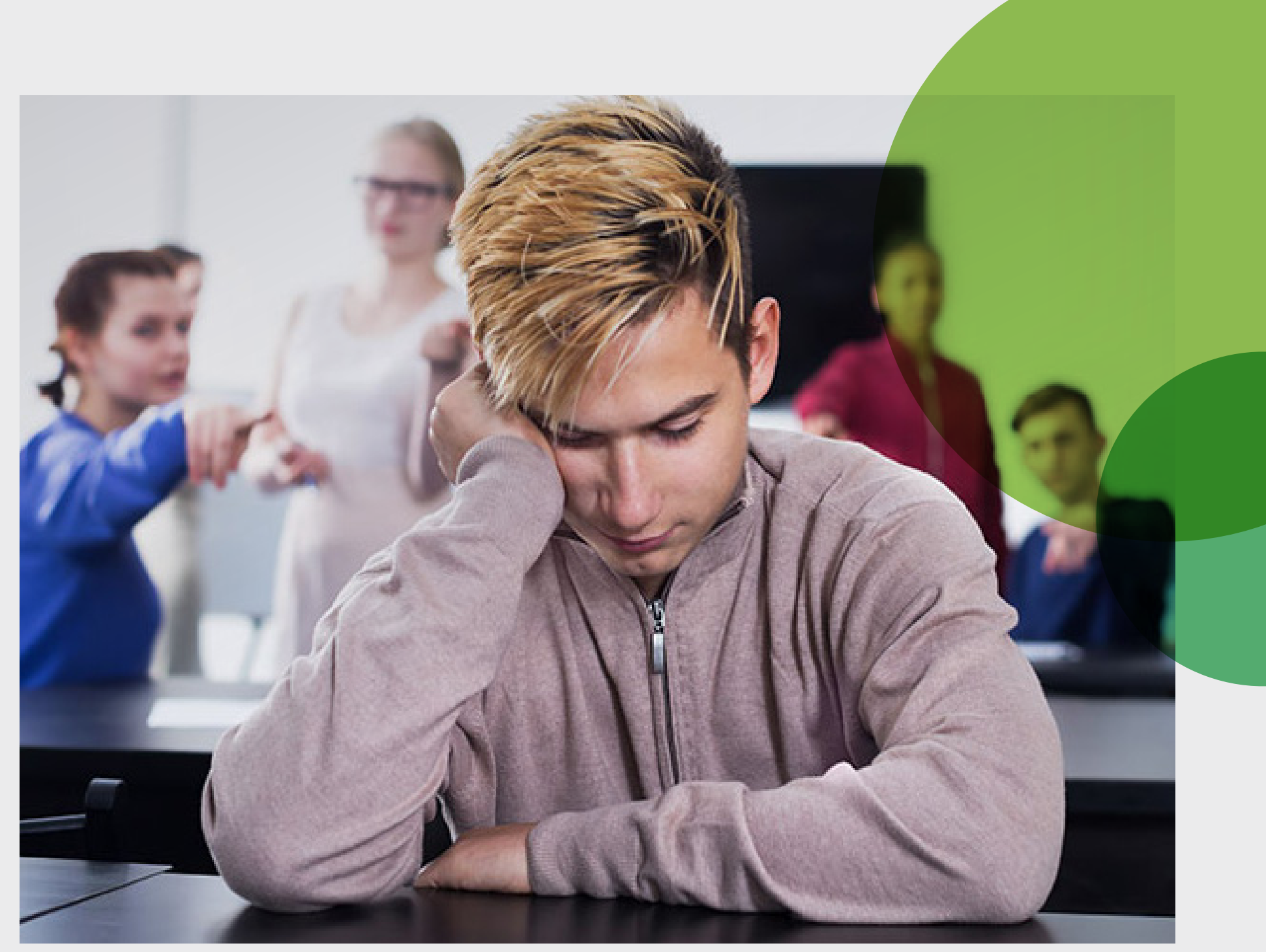

\title{
Challenging perspectives on Gilles de la Tourette Syndrome - evidence for a disorder of purposeful actions
}

By Christian Beste, Alexander Münchau

${ }^{1}$ Cognitive Neurophysiology, Department of Child and Adolescent Psychiatry, Faculty of Medicine of the TU Dresden, Germany

${ }^{2}$ Department of Pediatric and Adult Movement Disorders and Neuropsychiatry, Institute of Neurogenetics, Center for Brain, Behavior and Metabolism, University of Lübeck, Lübeck, Germany
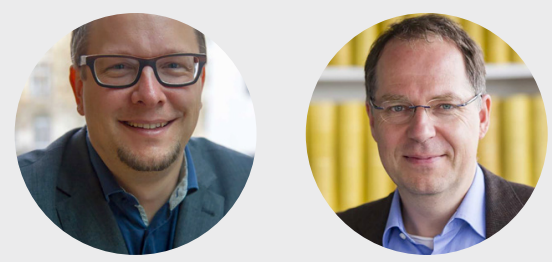

Gilles de la Tourette Syndrome (GTS) is a multifaceted neuropsychiatric developmental disorder with onset in childhood or adolescence. It is characterised by multiple motor and vocal tics that can cause considerable problems including social stigmatisation, low self-esteem and secondary comorbidity, particularly depression. Because these tics are perceived as a motor problem, GTS is typically considered a movement disorder. However, this view is beginning to change. In fact, there are many aspects of GTS, which do not fit to the concept of a straightforward movement disorder (Beste \& Münchau, 2018) these aspects include that (i) tics are associated with premonitory sensations, (ii) tics can be controlled and (iii) tics may be related to motor learning and habit formation. 
It seems that the symptoms of GTS do not or only incompletely fit to the characteristics of "classical" movement disorders. When taking a broader perspective, it seems more likely that tics represent an excess of purposeful actions and some kind of over-learned behaviour in the sense that the coupling between "perception" and "action" is altered in GTS. It has been suggested that GTS is associated with stronger connections between perception and actions and that the underlying deficit in GTS is the inability to weaken strong connections between perceptions and actions. In a recent study by Petruo et al. (2018), this novel perspective on GTS was examined for the first time; i.e. it was tested whether there are indeed stronger connections/bindings between perception and action in these patients.

To test this, the authors examined a group of children with GTS and healthy control children in a task where it is important to occasionally suppress responses. In other words, situation-appropriate behaviour was examined, which is a task relevant in daily life and sometimes difficult to accomplish by patients. In their experiment, the authors took advantage of the fact that especially multi-modal stimuli (i.e. stimuli that combine auditory and visual aspects) are very useful to guide actions. Critically, the authors also included conditions (situations) where the actions needed to be inhibited, but where the perceptual input was altered (i.e. the auditory aspect was lacking). This is critical for GTS patients, because these patients likely establish strong expectations that a particular action is preceded by the same input. If this expectation is violated, this will cause problems to guide actions. The authors showed that this is indeed the case in GTS patients. Moreover, they also showed what neural mechanisms underlie these processes. Their analyses revealed that altered action selection processes are indeed associated with the observed behavioural deficits. Attentional selection processes do not seem to be important for the observed problems in GTS. Taken together, the results provide first direct evidence for a new conceptual framework for GTS; i.e. that GTS might be conceived as a disorder of purposeful actions and alterations of the connection between perception and action. Notably, this finding has strong implications for the treatment of GTS.
Aside pharmacological treatments, also behavioural therapy (e.g. habit reversal training) is an important treatment approach that has been shown to ameliorate tics in GTS. The aim of this behavioural intervention is to replace tics with other movements that do not appear to be misplaced in context. The new concept that GTS might be a disorder of purposeful actions corroborates the usefulness of this approach and provides the missing theoretical background to this form of treatment. Importantly, the new framework and ways to test this framework in GTS will provide new diagnostic procedures. In addition, research in this domain will provide novel tools to examine different cognitive facets of GTS. Consequently, these new tools may prove useful in the diagnostic process of GTS and may also serve as new outcome parameters and endpoints for clinical studies in GTS. Currently, a study is underway that examines the effects of a habit reversal training using the abovementioned experiment. This is important because this study will test whether a major treatment approach is able to modify problems that are central for a new theoretical framework for GTS.

Referring to:

Beste, C., \& Münchau, A. (2018). Tics and Tourette syndrome - surplus of actions rather than disorder? Movement Disorders: Official Journal of the Movement Disorder Society, 33(2), 238-242. https://doi.org/10.1002/ mds.27244

Petruo, V., Bodmer, B., Brandt, V. C., Baumung, L., Roessner, V., Münchau, A., \& Beste, C. (2018). Altered perception-action binding modulates inhibitory control in Gilles de la Tourette syndrome. Journal of Child Psychology and Psychiatry, and Allied Disciplines. https://doi.org/10.1111/jcpp.12938 